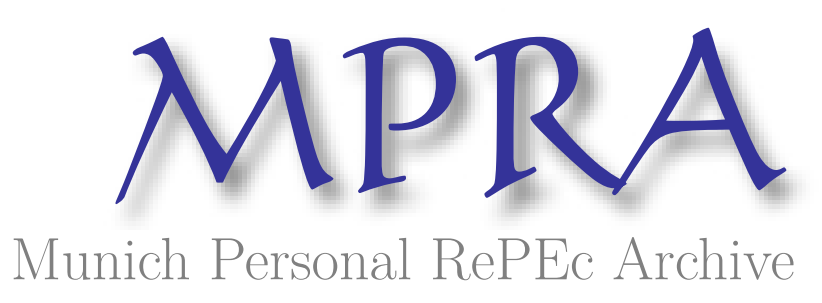

\title{
Necessary and sufficient conditions for a resolution of the social choice paradox
}

Chichilnisky, Graciela and Heal, Geoffrey

25 November 1979

Online at https://mpra.ub.uni-muenchen.de/8495/

MPRA Paper No. 8495, posted 28 Apr 2008 02:56 UTC 


\title{
Necessary and Sufficient Conditions for a Resolution of the Social Choice Paradox
}

\author{
Gracilila Chichilnisky
}

Deparment of Economics, Columbia University, New York, New York 10027

AND

Geoffrey Heal

Graduate School of Business, Columbia University, New York, New York 10027 and Department of Economics, University of Essex, Colchester CO4 $3 S Q$, England

Received November 25, 1979; revised October 20, 1981

\begin{abstract}
We present a restriction on the domain of individual preferences that is both necessary and sufficient for the existence of a social choice rule that is continuous, anonymous, and respects unanimity. The restriction is that the space of preferences be contractible. Contractibility admits a straightforward intuitive explanation, and is a generalisation of conditions such as single peakedness, value restrictedness and limited agreement, which were earlier shown to be sufficient for majority voting to be an acceptable rule. The only restriction on the number of individuals, is that it be finite and at least 2. Journal of Economic Literature Classification Numbers: $022,024,025$.
\end{abstract}

\section{INTRODUCTION}

Ever since Black $|3|$ and Arrow |1| first stated the social choice paradox clearly, considerable energy has been devoted to discovering conditions under which rational social choice is possible. This paper presents necessary and sufficient conditions, extending earlier sufficient conditions of Chichilnisky $|9|$.

One approach is to place restrictions on the types of preferences that can be held by individuals: technically, this amounts to specifying restrictions on the spaces of preferences. An example widely used in other branches of preference aggregation theory is the condition that preferences be homothetic. (For applications of this condition, see Chipman $|14|$ and Chichilnisky and Heal $|12|$.) This is also called a restricted domain approach. Another case is when any type of preference is in principle allowed, but there has to be a certain interrelationship between the preferences actually held by individuals. Existing conditions of these types 
are single peakedness (Black $|3|$ ) and value restrictedness (Sen and Pattanaik $|24|$ ). Conditions of this type are referred to as restricted profile conditions.

The suflicient conditions that have been given for rational outcomes under majority voting are all essentially combinatorial in nature (see Sen and Pattanaik $|24|)$. We use a different approach from that used by earlier writers, working within a framework introduced in Chichilnisky $|9|$. Because this approach views preferences as a collection of smooth indifference surfaces in a euclidean choice space, as is usual in the rest of economic theory, it may be more intuitively appealing than the combinatorial approach. Furthermore, it allows us to view the social choice problem as one of demonstrating the existence of a suitable continuous function from the Cartesian product of the preference space into itself. This in turn enables one to apply techniques which provide a characterization of the problem which is more comprehensive and yet more economical than any that was previously possible. It was demonstrated in Chichilnisky $19 \mid$ that an essential element of the social choice problem is that there is in general no map from the product of the preference space with itself, into the preference space, that is simultaneously continuous, anonymous and that respects unanimity.' This is the version of the problem that we work with: we give conditions for the existence of such a map. Note that the major difference form earlier work, apart from the mathematical tools used, is the replacement of the somewhat contentious axiom of independence of irrelevant alternatives by a more natural condition of continuity. ${ }^{2}$

Using this topological framework, we present conditions that are necessary' and sufficient for the existence of continuous anonymous rules that respect unanimity for any finite number of agents. The restricted domain condition is that the space of preferences be contractible ${ }^{3}$, i.e., that it admits a deformation to a point. The relationship between contractibility and aggregation was first discussed in Chichilnisky $|9|$, where the sufficiency of the condition of contractibility was proven. The equivalent restriction on preference profiles, is that the space of preference profiles be deformable onto the set of unanimous profiles. Although there are in the literature a number of conditions that are sufficient for the existence of specific social choice rules (in particular, majority voting), this is to the best of our knowledge the first statement of conditions that are both necessary and sufficient for a general resolution of the social choice paradox.

I It was also shown in Chichilnisky $|6|$ that the standard version of the problem, involving nondictatorship and the Pareto principle, has essentially the same mathematical structure.

${ }^{2}$ For a related exploraiion of continuity conditions in social choice problems, sec (oughlin and $\operatorname{Lin}|15|$.

I A topological space is contractible wheh it can be continuously deformed to one of its points, and therefore has the topological type of a point. 
Both interpretations of the result indicate that the social choice paradox has only "trivial" solutions. The space of preferences being contractible implies that up to a continuous deformation the space of preferences has only one point. Similarly, when considering the space of preference profiles, the condition is that the admissible space of profiles consists only of unanimous profiles up to a continuous deformation. The fact that only trivial solutions exist is consistent with the finding that for choice spaces of dimension at least 2 , the single peakedness condition of Black $|3|$ implies that all preferences are essentially identical, as shown in Kramer $|20|$.

We also demonstrate here that if the choice space is infinite dimensional and a Hilbert space, even with finitely many voters it is always possible with unrestricted domains to construct a continuous anonymous social choice rule which respects unanimity. This possibility theorem is clearly not robust, since for a choice space of any finite dimension, no matter how large, the usual impossibility theorem for finitely many voters holds. The fact that a possibility theorem holds when the dimension of the space is infinite, but does not for any finite dimension, is reminiscent of the fact that with a finitedimensional choice space, it is possible to construct a social choice rule when the number of agents is infinite, whereas once again the usual impossibility results hold for any finite set of agents. This fact was first noted by Fishburn $|17|$, and later by Brown $|4|$, and Kirman and Sonderman $|19|:$ these results were extended to continuous anonymous rules in Chichilnisky and Heal |13|.

It is natural to inquire into the relationship between our sets of necessary and sufficient conditions, and earlier sufficient conditions (such as those of Sen and Pattanaik $|24|$ ) for a rational outcome under majority rule, since these rules satisfy the anonymity condition and respect unanimity. In fact, such a comparison is not straightforward. This is because our conditions apply to the existence of social choice rules in general, whereas those earlier conditions relate only to majority rules. Furthermore, here we require that a social choice rule be continuous, so that small changes in the preferences (or a small error in the reporting of preferences) leads only to small changes in the outcome. Continuity was not required in the earlier literature; indeed, their framework did not permit the discussion of such a condition. Now it was shown in Chichilnisky $|10|$ that even rather weak forms of majority rules (decisive majorities) are in general not continuous, so that the rules for which the earlier conditions were devised, do not in fact meet our assumptions. However, it is clear that our conditions are necessary for the existence of satisfactory continuous majority rules, and in spite of the differences in approach some of the earlier conditions appear to be special cases of ours. As a demonstration of this is rather complex, we leave it to a separate paper.

It seems useful to discuss briefly the applicability of the necessary and 
sufficient conditions given here, to the version of the social choice paradox involving nondictatorship and the Pareto condition.

The continuity assumption, as mentioned, takes the role of the axiom of independence of irrelevant alternatives. The condition of respect of unanimity that we require (that if all preferences are identical over all choices, then the social preference is the same as that of the individuals), is clearly weaker than the Pareto condition, whereas the anonymity condition that we require is stronger then the nondictatorship condition. Anonymity requires complete symmetry in the treatment of voters, whereas nondictatorship merely eliminates the most extreme forms of asymmetry. Therefore, the necessary and sufficient conditions that we give here may not apply to the existence of a continuous Pareto nondictatorial social choice rule, although we conjecture in view of the results of Chichilnisky $|6|$ that this is the case under certain regularity conditions.

In the next section, we introduce the framework within which we shall be working and present the relevant notation and definitions. In Section 3, we discuss in intuitive terms the main results of the paper, giving examples both of preference domains that are contractible, and of the type of social choice rule then admitted. The fourth and final section contains a formal statement of the theorems; some of the proofs are in the Appendix.

\section{Notation AND Definitions}

Let $Y$ be the choice space, such as a unit cube in $R^{n}$, denoted $I^{n}$, or the positive orthant of $R^{n}, R^{n+}$. Since our framework is topological, it suffices to consider a space which is homeomorphic to $I^{n}$ : to simplify matters we choose the closed unit ball $\boldsymbol{B}^{n}$, a manifold with boundary.

A preference $p$ on $Y$ is a family of smooth indifference surfaces: formally, it is defined by giving for each choice $y$ in $Y$ a preferred direction, or, equivalently, the normal to the indifference surface at $y$, a vector denoted $p(y)$. As is usual in social choice theory (e.g., Arrow $|1|$ ), preferences are ordinal, and intensities of preferences are not considered. We therefore normalize the vector fields that give our preferences to be of unit length, i.e., $\|p(y)\|=1$ for all $y{ }^{4}$ A preference is thus a map $y \rightarrow p(y)$ from choices $y$ into the tangent space of $Y, T(Y)$, such that for each $y, p(y)$ is in the tangent space of $Y$ at the choice $y$. Such a map is called a vector field on $Y$. We topologize the spaces $Y$ and $T(Y)$ as usual, and we assume that the

- The fact that the vector field can be normalized to be of unit length, implies that it is nowhere zero in the interior of the choice space. In the remark following Corollary 2 below, we indicate how our results can be extended to certain spaces of preferences which admit sattiation. A general discussion of social choice problems with vanishing gradients within the present framework is contained in Chichilnisky $|7|$. 
preferences are defined by continuously differentiable locally integrable vector fields, i.e., that $p(y)$ is locally the gradient of a real-valued utility function on $Y$. The space $P$ of all such locally integrable preferences is characterized within the space of all $C^{\prime}$ vector fields on $Y, V(Y)$, by the Frobenius integrability conditions; $V(Y)$ is given the $C^{1}$ topology. ${ }^{5.6}$ The Frobenius conditions and the normalization assumption $\left\|p\left(y^{\prime}\right)\right\|=1$ are both. closed conditions, so that the space $\boldsymbol{P}$ endowed with the topology inherited from $V(Y)$ is a complete space since it is a closed subspace of the space of vector fields $V(Y)$. The space $V(Y)$ is infinite dimensional, and the space $P$ contains infinite-dimensional manifolds; this is shown in Chichilnisky $|5|$.

We assume that there are $k$ voters indexed by $i=1, \ldots, k$. $k$ is finite. A profile of $k$ voters' preferences is given by a sequence $\left\{p_{i}\right\}=\left(p_{1}, p_{2}, \ldots, p_{k}\right)$. We shall denote the space of profiles $L$; this is a subset of the product of the space $P$ with itself $k$ times. We denote this product space $X P_{i} ; P_{i}$ denotes the space of preferences of the $i$ th voter and thus $P_{i}=P$ for all $i$, so that $X P_{i}=X P$.

A social aggregation rule or social choice rule is a map from profiles to social preferences in $P$. Therefore,

$$
\phi: L=X P \rightarrow P .
$$

Note that the social choice rule $\phi$ has as its arguments preferences $p_{i}$, which are vector fields over the entire choice space. It is not assumed to act "pointwise" by combining at each choice the individual preferences at that choice to give a social preference at that choice. The social preference at a point $y$ may in principle depend on individual preferences over their entire domain. Of course, "pointwise" rules come within the scope of our results.

A social choice rule $\phi$ is said to be anonymous if for any permutation $\left\{m_{1}, \ldots, m_{k}\right\}$ of the set of integers $\{1, \ldots, k\}$,

$$
\phi\left(p_{1}, p_{2}, \ldots, p_{k}\right)=\phi\left(p_{m_{1}}, \ldots, p_{m_{k}}\right) .
$$

The outcome of the social choice rule thus depends only on the set of preferences of the individuals, and not on who holds which preference. This is an equal treatment condition: it implies that there is no one individual whose preference determines the social choice, so that in particular the rule

'We give $V$ the $C$ ' topology in order to obtain a nice topological structure on $V$ : any topology on $V$ that restricted to linear preferences coincides with the convergence of vectors in $R^{n}$, will also give our result.

- The Frobenius integrability conditions are usually given by a set of partial differential equations. They are necessary (but not sufficient) for a vector field to be the gradient of a real valued function. For a discussion of these conditions see, for instance, Debreu $|16|$. 
is nondictatorial. The social choice rule is said to respect unanimity if

$$
\phi(p, p, \ldots, p)=p, \quad \text { for all } \quad p \in P
$$

i.e., if all individuals have identical preferences over all choices, then the social choice rule yields that preference as an outcome. This is clearly a very natural requirement. Note that this does not imply that if all individuals prefer alternative $x$ to alternative $y$, then society prefers $x$ to $y$. It is therefore weaker than the Pareto condition. It was shown in Chichilnisky $|9|$ that with unrestricted domains of preferences there is no social choice rule $\phi$ :

$$
\phi: X P \rightarrow P,
$$

which is simultaneously continuous, anonymous, and respects unanimity. The space $X P$ has the natural product topology inherited from that of the space $P$, and continuity of the social choice rule $\phi$ is defined with respect to this topology on XP. Continuity of the rule $\phi$ and of the preferences implies that the social preference at a point $y \in Y$ is continuous in profiles and choices. Although we are not requiring the axiom of independence of irrelevant alternatives, these continuity properties will ensure that a social choice rule satisfies a related global independence condition, see Chichilnisky and Heal $|13|$.

A topological space $X$ is contractible if there exists a continuous function $f$ and a point $x_{0} \in X$ with

$$
f: X \times|0,1| \rightarrow X,
$$

such that

$$
f(x, 1)=x, \quad \text { for all } \quad x \in X
$$

and

$$
f(x, 0)=x_{0}, \quad \text { for all } \quad x \in X
$$

In more intuitive terms, a contractible space is one that has no "holes" in it, and can consequently be contracted continuously through itself into one of its points. Any convex space is contractible, as is the unit disk; the circle is not contractible, nor is the torus.

A topological space $X$ is connected when it does not contain two subsets $A$ and $B$ such that both $A$ and $B$ are open and closed in $X, A \cap B=\phi$, and $X=A \cup B . Y \subset X$ is called a connected component of $X$ if $Y$ is connected, and there is no connected subset $Z$ of $X$ such that $Y \varsubsetneqq Z$. 


\section{Discussion and Examples}

We shall give below two necessary and sufficient conditions for the existence of a social choice rule, one relating to properties of spaces of profiles, and the other to domain restrictions. In both cases, the essential point is that the domain of individual preferences, $P$, should be contractible. In this case, and only in this case, we can always construct for any finite number of voters a social choice rule which is continuous, anonymous, and respects unanimity. In this section, we discuss in general and intuitive terms the interpretation of these results, and illustrate them with particular examples of contractible domains and of the admissible social choice rules. (For further illustrations of the concept of contractibility and of its relationship with other more familiar concepts such as convexity, see Heal |18|.)

In Theorem 1 below, we follow the restricted domain approach, and show that a necessary and sufficient condition for the existence of a social choice rule for any finite number of voters is that the space of preferences be contractible. Equivalently, we show in Corollary $I$ that if attention is directed to the study of spaces of preference profiles, the necessary and sufficient condition is that the profile space admits a rctraction ${ }^{7}$ to the preference space.

Consider first the restricted domain condition. In this case, the condition is that the space of preferences be contractible, which means that it can be continuously deformed to a point. A point represents a single preference, so this is a requirement that the preferences in the space of preferences are sufficiently similar that they can all be simultaneously continuously deformed to a single preference. Therefore, this is a requirement of unanimity up to a continuous deformation.

Next consider the condition on profiles and its implication that the profile space and the preference space be of the same topological type. This means that one can be continuously deformed into another, i.e., that the set of all possible profiles can be continuously deformed into the set of all possible preferences. Thus any element of the former, i.e., any profile, can be continuously deformed into an object in the latter, a single preference. In other words, within any admissible profile there must be sufficient agreement that the preferences in that profile can be continuously deformed into one single preference. This is again a requirement of unanimity up to a continuous deformation in any admissible profile.

We see then that our two sets of necessary and sufficient conditions are in essence the same. In both cases, what is required is that any preference

1 A retraction $r$ from $X$ onto a subset $A c^{-} X$ is a continuous map $r: X+A$ that when restricted to $A$ is the identity. Intuitively, $r$ pulls $X$ onto $A \subset X$. 
profile that may arise, exhibits sufficient agreement that the preferences in it can be continuously deformed to a single preference. What one might call "Iopological unanimity" is a general necessary and sufficient condition for the existence of appropriate aggregation rules with any linite number of agents.

It should of course be emphasized that "topological unanimity" is much weaker than unanimity. A set may be very large and still be continuously deformable to a point-for example, a cone in Euclidean $n$ space is contractible. The role of the "topological unanimity" condition is to limit the types of disagreement or of variation in preferences that can be accepted. The examples below make this point clear. Limiting the nature and extent of possible disagreement is clearly the effect of many of the conditions already cited in the literature as sufficient for a rational outcome under majority voting-for example, single peakedness, limited agreement, and valued restriction. Kramer $|20|$ explores the implications of these conditions, and shows how they are limiting the range of variation or disagreement in preferences. Indeed, he shows that for a choice space of dimension at least three, single peakedness implies unanimity, and thus that the space of preferences is a point.

It is interesting to relate these conclusions to a remark made by Arrow | 1 , p. 831. Speaking of Black's single-peakedness condition, he commented that it did at least "show that the condition of unanimity is mathematically unnecessary for the existence of a social welfare function." Kramer's result shows that in the case of single peakedness, this optimism was misplaced. More generally, our results show that a revised and greatly weakened concept of unanimity provides a necessary and sufficient condition.

We now turn to a discussion of the implications of Theorem 1, which gives contractibility as a necessary and sufficient restriction on the preference domain. This tells us that restrictions must be placed on the types of preference admitted which ensure that the space of preferences is topologically trivial, i.e., that it has the topological characteristics of a point. To clarify this, we consider its implications when we restrict our attention to preferences at one choice.

Within our framework, if we restrict our attention to preferences at a choice $y$ in $Y$, then a preference is fully characterized by the normalized gradient vector $p_{i}(y)$ giving individual $i$ s preferred direction at $y$. If there are no restrictions on individual preferences, then this normalized gradient vector may take any direction, so the set of possible preferences at the point $y$ is isomorphic to the set of points on the unit sphere centered at $y$. This is, denoted by $S^{n}$ ', the $(n-1)$-dimensional sphere in $R^{n}$. A restriction on individual preferences now takes the form of the specification of a subset of $S^{n}$ ' within which the normalized gradient vector must lie.

If there is no restriction on individual preferences, then the space of 
individual preferences at a point is $S^{n}$ ', and is therefore not contractible. Hence, no social choice rule will exist. A listing of individual preferences at a point is then an element of the product of the sphere $S^{n-1}$ with itself $k$ times.

If, however, there is a convex cone of directions $C$, no matter how small, which no agent may have as most preferred directions, then the space of preferences becomes the sphere $S^{n-1}$ minus its intersection with a cone, which is contractible. In this case, a social choice rule exists. It should be noted that the size of the cone $C$ of excluded directions (measured for instance in euclidean distance) is a property which is not invariant under different units of measurement. It is therefore desirable that the results do not depend on the size of such cones, as, indeed, they do not.

The above gives a sufficient condition for the contractibility of the space of preferences at each choice. If this condition holds at every choice, and if the contraction to be applied at each point varies continuously with that point, and satisfies certain other regularity conditions, then the overall space of preferences $P$ will be contractible. In intuitive terms, what is required to move from local to global contractibility is that the cone of directions excluded at any point should vary "smoothly" with that point, as shown in Fig. 1.

We give some simple examples to illustrate this idea.

Exлmple: 1. If the excluded directions comprise the same convex cone at every point in the choice space, then the space of preferences is contractible. Thus if there is some direction such that everyone agrees that their most preferred direction is bounded away from this, and all other directions are permitted, a social choice rule exists. In particular, if the space of preferences consists of all monotone and convex preferences, then a social choice rule exists. This appears at first sight to cover the case of the usual general equilibrium model, but in fact this is not true. Under the usual

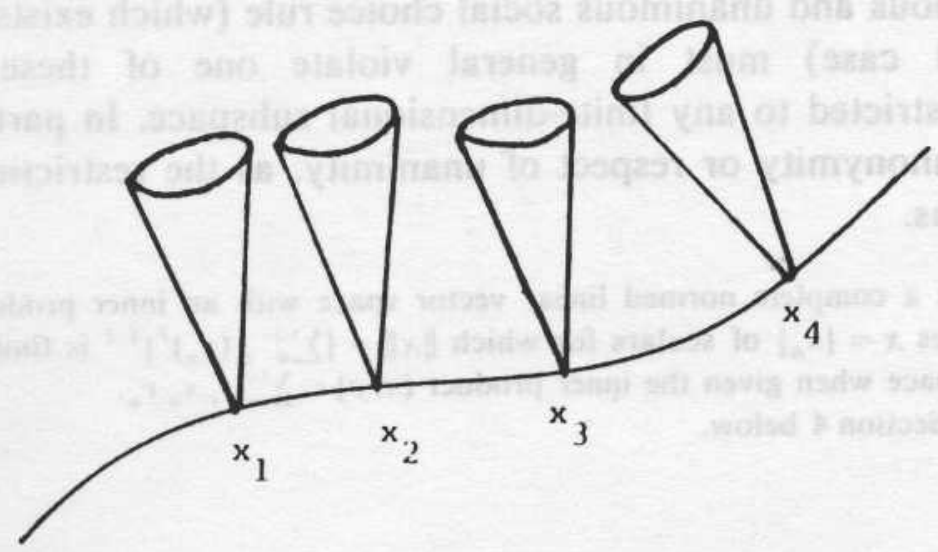

Fic;. I. Cones of excluded directions at points $x_{1}, x_{2}, \ldots$. 
assumptions, the relevant space over which social choices have to be made in this model, is the generalised Edgeworth box, on which individual preferences may be opposed, and are clearly not a monotone family.

Note that while Arrow's version of the social choice paradox (Arrow |1|) cannot be resolved with convex monotone preferences over the choice space $B^{n}$, it can be resolved if preferences are convex and monotone over every triple of alternatives. The axiom of independence makes such a triple the basic unit in Arrow's framework: see Chichilnisky |7|.

Example 2. Suppose that preferences are linear, so that their gradients are constant and the space of preferences is identical with the sphere. Then in this case, the space of preferences is contractible if it consists of all linear preferences that are bounded away from one preference.

Example 3. Consider the case where there are a finite number of agents. but the choice space for each is a Hilbert space of countably infinite dimension. ${ }^{8}$ The space of linear preferences is then the unit sphere which in such spaces is homeomorphic to the whole space (see Bessaga and Pelczynski $\mid 2$, Corollary 5.1, p. $109 \mid$, and Kuiper $|21|)$. In particular, it is contractible and furthermore, it is also homeomorphic to a convex set, i.e., it is in a one to one bicontinuous correspondence with a convex set. Note first that Theorem 1 applies because the sphere in a Hilbert space is a parafinite $C W$ complex. ${ }^{9}$ In fact, this case is particularly simple: we can alway's construct a continuous anonymous social choice rule that respects unanimity simply by taking convex addition composed with the homeomorphism, as shown below.

The case of a choice space of countably infinite dimension, is one that has not previously been noted, and therefore merits some further comment. The result quoted on the contractibility of the unit sphere in Hilbert spaces is in fact counterintuitive and it is the basis for the resolution of the paradox in these cases.

It is also counterintuitive to have a possibility result only when the dimension of the choice space is infinite-we noted a similarly counterintuitive result in the case of infinitely many agents. It is clear that any continuous anonymous and unanimous social choice rule (which exists in the infinite-dimensional case) must in general violate one of these three conditions when restricted to any finite-dimensional subspace. In particular, they must violate anonymity or respect of unanimity, as the restriction will surely be continuous.

" A Hilbert space is a complete normed linear vector space with an inner product. The space $l_{2}$ of all sequences $x=\left|x_{n}\right|$ of scalars for which $\|x\|=12_{n}^{\cdots},\left.\left(x_{n}\right)^{\prime}\right|^{1}$ is linite. is an example of a Hilbert space when given the inner product $(x, y)=2_{n}^{\prime \prime}, x_{n} y_{n}$.

$\checkmark$ This is defined in Section 4 below. 
Although this result on infinite-dimensional spaces is counterintuitive, it is certainly not without economic interest. There are many economic objecte between which social choices have to be made, which are naturally of infinite dimension. Examples are optimal growth programmes, optimal resource depletion profiles, and optimal income tax schedules. All of these are most naturally modelled as infinite-dimensional objects, so that the fact that the social choice problem can be resolved in the infinite-dimensional case is of considerable relevance for growth theory and tax theory.

Example 4. It should be emphasized that a contractible preference space need not be constructed by restricting the set of directions that preference gradients can take at each choice. A construction in Chichilnisky $|5|$ considers preference families such that there is a one-dimensional neat ${ }^{10}$ submanifold of the choice space along which all members of the family are increasing. It is shown there that such families are contractible, even though there may be regions of the choice space at which all possible gradients are assumed. Figure 2 illustrates this construction: the curve $a b$ is a onedimensional neat submanifold of the unit cube along which all the preferences are increasing.

ExAmple 5. It may be helpful, finally, to give examples of preference spaces which are not contractible. One is the whole space $P$ (see

b

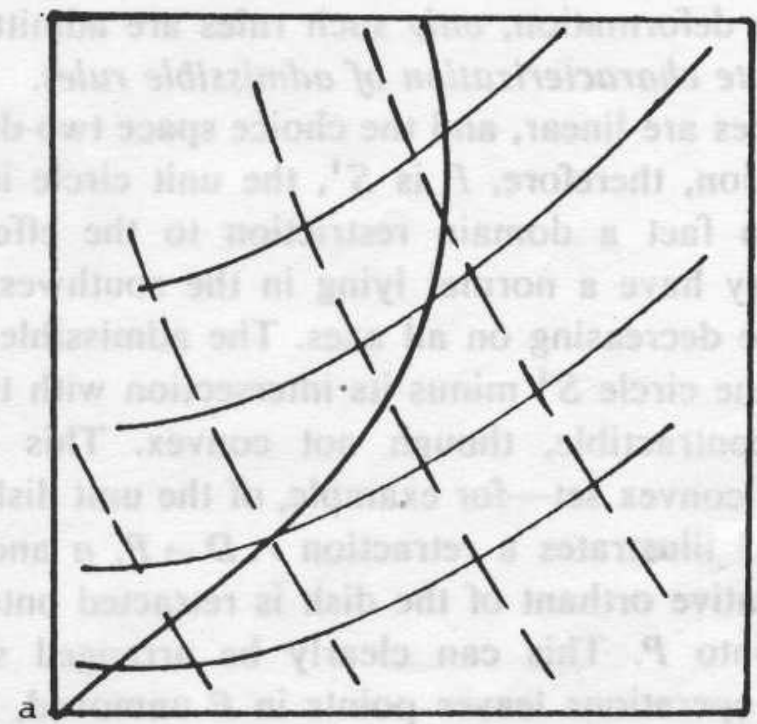

Fici. 2. $a b$ is a neat one dimensional submanifold of the choice space. A family of preferences is contractible if all members are increasing along $a b$.

110 A neat submanifold $B$ of a manifold $A$ is a submanifold such that ${ }^{\prime} B C$ C $A$, i.e., the boundaries of $B$ are contained in those of $A$. 


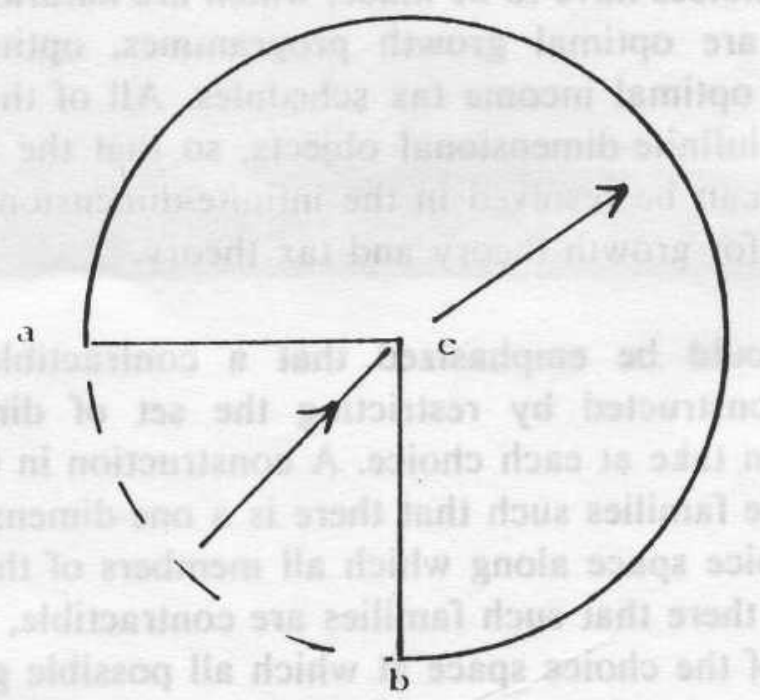

Fic;. 3. A two-stage retraction of the disk $D$ onto $P$.

Chichilnisky $|9|)$. It is also shown in Chichilnisky $|11|$ that under standard assumptions the space of von Neumann-Morgenstern utilities over a finite set of alternatives, is not a contractible space.

Finally, we give an example of the kind of social choice rule which our theorems admit. As the sufficiency proof of Theorem I will indicate, convex averaging rules, or deformations of these, are admitted. Theorem 2 shows that, up to a continuous deformation, only such rules are admitted, so that we have in fact a complete characterization of admissible rules.

Suppose that preferences are linear, and the choice space two-dimensional. With no domain restriction, therefore, $P$ is $S^{\prime}$, the unit circle in $R^{2}$. Now suppose that there is in fact a domain restriction to the effect that no individual preference may have a normal lying in the southwest quadrant, i.e., no preference can be decreasing on all axes. The admissible domain of preferences is therefore the circle $S^{1}$ minus its intersection with the negative orthant: it is clearly contractible, though not convex. This domain is, however, a retract " of a convex set-for example, of the unit disk $D^{\prime}$ ' whose boundary is $S^{\prime}$. Figure 3 illustrates a retraction $r: D \rightarrow P$. $a$ and $b$ are the ends of $P$. First the negative orthant of the disk is retracted onto ach, then $a c b$ is "pulled back" onto $P$. This can clearly be arranged so that the composition of the two operations leaves points in $P$ unmoved, as required by a retraction.

We can now define the following social choice rule. Consider a $k$-tuple of preferences $p_{1}, \ldots, p_{k}$ with $p_{i} \in P$. Firstly, compute the mean,

$$
p=\frac{1}{k} \frac{1}{i} p_{i}
$$

" $A$ is a retract of $B$ if there is a retraction (see note 7 ) of $B$ onto $A$. See also the definition in Section 4 below. 
If $p \in P$, then $p$ is the social preference. Otherwise, $p$ will certainly be in $D)^{\prime}$. In this case, apply to $p$ the retraction $r$ defined above, leading to a point in $P$. This is now the social preference.

Note that in the example given here, $P$ is not only a retraction of a convex set: it is homeomorphic to a convex set (namely, a closed connected interval of $R^{\prime}$ ). In this case we could in fact have used the much simpler construction of averaging in the convex set, and composing this with the inverse of the homeomorphism. However, not all contractible spaces are homeomorphic to convex sets, so that this argument cannot be used in the proof of Theorem 1.

\section{Necessary and SUfFicient Conditions}

In this section we give formal statements of the theorems alluded to above. Some of the proofs are also given here, with the remainder in the Appendix. First, however, we need to introduce some further topological terms and concepts.

In the following, the space of preferences, denoted by $P$, is assumed to be a parafinite $\mathrm{CW}$ complex with finitely many connected components, whose convex hull $k(P)$ has the same characteristics. A $C W$ complex is a topological space that can be constructed inductively by adding $n$-cells is an appropriate fashion; an $n$-cell is homeomorphic to an $n$ disk in $R^{n}$ (see Spanier $\mid 25$, p. $401 \mid)$. We call a $C W$ complex parafinite when it has a finite number of cells of each dimension $n \geqslant 0$. In Corollary 2 below it is shown that the connectedness of the space is not essential, as analogous results follow if the space is not connected, but each connected component is a parafinite $\mathrm{CW}$ complex with the above properties.

This is a very general specification; it includes the case where $P$ is the $n$th sphere $S^{n}$, when $P$ is an $n$-dimensional smooth manifold, or when $P$ is a polyhedron, or any space homeomorphic to a polyhedron. Certain infinitedimensional spaces of preferences are also included, such as the unit sphere in a Hilbert space as in Example 3. This can be constructed by the inductive process of adding two $n$-cells to $S^{n-1}$ for all $n \geqslant 2$, and taking the union. This condition that the space of preferences be a parafinite $C W$ complex is used in the proof of Theorem 1 below. In particular, being a $\mathrm{CW}$ complex is required to ensure that the space of preferences, which is connected and has all homotopy groups zero, will be contractible, see, e.g., Spanier $\mid 25$, 7.6.24|. ${ }^{12}$ This may not be true with certain pathological spaces such as a

12 Corollary 24, Chapter 7, Section 6, of Spanier $|25|$ proves that a map hetween $\mathrm{CW}^{\prime}$ complex is a weak homotopy equivalence if and only if it is a homotopy equivalence. By definition of a weak homotopy equivalence (Spanier $|25, \mathrm{p} .404|$, the constant map $f: P \cdots P_{0}$. where $P$ is the space of preferences and $p_{0}$ is a point in $P$, will induce a one to one correspon dence between path components of $P$ and of $\left|p_{0}\right|$. and an isomorphism in the homotopy 
“double comb" space, see, e.g., Maunder 123, No. 7.5, p. 301|. While our proof does not apply to such spaces, it is an open question whether a noncontractible preference space with zero homology may admit a well behaved social choice rule.

A space $A$ is said to be a deformation of $X$, or, equivalently, $X$ is deformable into $A$, if there exists a continuous map

$$
D: X \times|0,1| \rightarrow X
$$

such that

$$
D(x, 0)=x \forall x \text { in } X,
$$

and

$$
D(X, 1)=\mathcal{A} \text {. }
$$

A space is contractible if it is deformable into one of its points. A retraction $r$ from $X$ onto $A \subset X$ is a continuous map $r: X \rightarrow A$ that restricted to $A$ is the identity, i.e., $r / A=\mathrm{id}_{A}$. In this case, the space $A$ is said to be a retract of $X$. The space $A$ is a deformation retract of $X$ if $A$ is a deformation and a retract of $X$.

Consider two continuous maps $f, g$ from a topological space $X$ into another $Y$. We say that $f$ and $g$ are homotopic if there exists a continuous maps $G$ such that

$$
\begin{aligned}
& G: X \times|0,1| \rightarrow Y, \\
& G(x, 0)=f(x) \quad \text { for } x \text { in } X, \\
& G(x, 1)=g(x) \quad \text { for all } x \text { in } X .
\end{aligned}
$$

$f$ and $g$ are thus homotopic if there is a continuously parametrised family of maps, parametrised by the unit interval, linking $\int$ and $g$. Intuitively, the map $f$ can be continuously deformed into $g$. We shall say that two social choice rules are equivalent if they are homotopic as maps.

In this section, we state two results giving necessary and sufficient conditions for the existence of a continuous, anonymous social choice rule that respects unanimity for any finite number $k$ of voters, $k \geqslant 2$. Theorem 1 extends the results in Chichilnisky $|9|$ to show that contractibility is a necessary as well as sufficient condition. Only the proof of sulficiency in

groups $\pi_{4}(P)$ and $\pi_{4}\left(p_{0}\right)$ for all $q \geqslant 1$, if $P$ is path connected and all homotopy groups of $P$. $(\pi,(P) \vee i)$ are zero. Therefore, Corollary 24 implies that when $P$ is a $C W$ complex with $\pi_{i}(P)=0$ for all $i, P$ is homotopically equivalent to $p_{0}$, and, therefore, $P$ is contractible. For another proof that a $\mathrm{CW}$ complex with all its homotopy groups zero is contractible, see Maunder 123, p. 329, Corollary 8.3.11|. 
Theorem I is given in this section, as this is relatively nontechnical. The remaining proofs are given in the Appendix. The sufficiency proof in Theorem I provides a characterisation of the kind of rule admitted.

In addition, we prove a theorem which establishes that up to a continuous deformation, all the continuous, anonymous social choice rules which respect unanimity (which exist only when the preference space is contractible) are identical to an appropriate generalisation of the example discussed in Section 3. That is to say, they are homotopic to rules constructed by convex averaging on a convex space, and then composing this operation with one which sends the convex space into the contractible space.

TH:овем 1. Let the space of preference profiles be the $k$-fold Cartesian product of the space of preferences, $L=X P$, so that all logically possible combinations of preferences are admitted. Then a necessary and sufficient condition for the existence of a social choice rule which is continuous, anonymous, and respects unanimity for all $k \geqslant 2$ is that the space of preferences $P$ be contractible, i.e., that up to a continuous deformation, all preferences are identical.

Proof. Let $P$ be a contractible space, $P \subset V(Y)$. Suppose first that $P$ is also convex. By definition, in a convex space averaging is always possible. It follows immediately that a continuous anonymous rule $\phi$ respecting unanimity exists, for instance

$$
\phi\left(p_{1}, \ldots, p_{k}\right)=\frac{1}{k} \leq p_{i}
$$

where $\sum$ denotes the vector sum in the space of $C^{1}$ vector fields $V(Y)$ induced by summing vectors at each point. The social preference is just the mean of the individual preferences. In fact we shall now show that, even if $P$ is not convex, a similar construction can still be used. Note first that since $\boldsymbol{P}$ is a contractible $C W$ complex, it is therefore a retract of the convex set $k(P)$, because the identity map

$$
i: P \rightarrow P
$$

can always in this case be extended to a continuous map $r: k(P) \rightarrow P$ by results of obstruction theory (see, e.g., Spanier |25, Chapt. 8, 8.4.1|). Therefore, since $r$ extends $i, r / P=\mathrm{id}_{p}$. By definition, $r$ is therefore a retraction from $k(P)$ onto $P$. It follows that the composite map

$$
\phi\left(p_{1}, \ldots, p_{k}\right)=r \circ\left(\frac{1}{k} \leq p_{i}\right)
$$


defines a continuous anonymous rule that respects unanimity. Therefore, to construct the social preferences, we perform an averaging operation on the convex space of which $P$ is a retract, and then compose this with the retraction on $P$. This completes the proof of sufficiency. The proof of necessity is given in the Appendix.

We shall now give a characterization of contractible spaces of preferences.

COROllary 1. A necessary and sufficient condition for the space of preferences $P$ to be contractible is that the space of preference profiles $L=X P$ admits a deformation retract onto the space of unanimous profiles. Therefore, with restricted domains, a solution exists for all $k \geqslant 2$ if and only if all preferences profiles are topologically equivalent to unanimous profiles.

The proof of this corollary is given in the Appendix.

THEOREM 2. Let the preference space $\boldsymbol{P}$ be contractible. Then any continuous anonymous social choice rule that respects unanimity, is equivalent to a rule $f$ constructed as follows. Take a convex space $C$ of which $P$ is a retract, with $r$ the retraction, $r: C \rightarrow P$. Then define a convex averaging rule on $C$, and let $f$ be the composition of this with $r$.

Proof. The existence of a convex space $C$ and a retraction $r: C \rightarrow P$ is assured by the arguments in the proof of sufficiency in Theorem 1. These arguments also demonstrate that there always exists at least one continuous anonymous social choice rule that respects unanimity and is constructed as specified in the theorem. We therefore only have to prove that any other such rule is homotopic to this. But this is an immediate consequence of the following observation:

Let $X$ and $Y$ be two topological spaces, with $Y$ contractible. Then any two continuous functions from $X$ to $Y$ are homotopic, see Spanier $|25,1.3 .7|$.

The next corollary extends the above results, and in particular those of Theorem 1, to disconnected spaces of preferences. We assume now that each connected component of the space of preferences is a parafinite $\mathrm{CW}$ complex, and that $\boldsymbol{P}$ has at most finitely many components.

CORotlary 2. Let the space of preference profiles be the $k$-fold Cartesian product of the space $P$ of preferences. Then a necessary and sufficient condition for the existence of a social choice rule which is continuous, anonymous, and respects unanimity for all $k \geqslant 2$, is that each connected component of $\boldsymbol{P}$ be contractible.

We prove necessity first. Let $\phi: P^{k} \rightarrow P$ satisfy all the conditions, then if $C$ is a connected component of $P$, continuity and respect unanimity imply $\dot{o} / C^{k}$ maps $C^{k}$ into $C$. Since $\phi / C^{k}$ obviously is a continuous anonymous map 
respecting unanimity, and $C$ is a connected parafinite $C W$ complex, Theorem I applies, and $C$ must be contractible.

Next we prove sufficiency. Let $P=\cup C_{a}, C_{a}$ the connected components of $P$. For each $C_{n}$, we can define a continuous map $\phi_{a}:\left(C_{n}\right)^{k} \rightarrow C$ respecting unanimity and anonymity, by Theorem 1 , since $C_{\alpha}$ is contractible.

Consider now any profile $\left(p_{1}, \ldots, p_{k}\right) \in P^{k}$. If all $p_{i}$ 's are in one component, say $C_{B}$, then define $\phi\left(p_{1}, \ldots, p_{k}\right)=\phi_{B}\left(p_{1}, \ldots, p_{k}\right)$. If instead, there exist in the profile $\left(p_{1}, \ldots, p_{k}\right)$ preferences which belong to different components, then define $\phi\left(p_{1}, \ldots, p_{k}\right)=\phi_{a}\left(p_{1}^{\prime}, \ldots, p_{k}^{\prime}\right)$, where $\alpha=\min \left(\alpha_{1}, \ldots, \alpha_{k}\right), p_{i} \in C_{a_{i}} ; p_{i}^{\prime}=p_{i}$ if $p_{i} \in C_{a}$, and $p_{i}^{\prime}=p_{a}$ if $p_{i} \notin C_{a}$. This rule satisfies all conditions.

Remark. Note that Corollary 2 enables us to apply Theorem 1 to certain spaces of preferences which admit satiation. For example, the space of linear preferences on $R^{n}$, including the preference which is indifferent between all alternatives, can be represented as the union of the unit sphere in $R^{n}$ and the origin. For further examples of spaces of preferences admitting satiation which come within this framework, see Chichilnisky $|7|$.

\section{APPENDIX: Proof OF the Necessity OF CONTRACTIBILITY IN THEOREM I}

The strategy of the proof is to show that the conditions of anonymity and unanimity together imply that any element of any homotopy group of $P$ is divisible by any arbitrary integer to yield a member of the group, thus implying that all such elements are zero. Because $P$ is a $C W$ complex, the fact that all homotopy groups are zero is then shown to imply that $P$ is contractible.

Let $\phi: P^{k} \rightarrow P$ be a continuous anonymous social choice rule respecting unanimity. $\phi$ defines a map $\phi^{*}$ at the homotopy level; for each $i \geqslant 1$, letting $\pi_{i}(P)$ be the $i$ th homotopy group of $P$,

$$
\phi^{*}: \pi_{i}\left((P)^{k}\right) \rightarrow \pi_{i}(P) .
$$

Consider first the case where $i \geqslant 2$. Then $\pi_{i}(P)$ is always abelian (see, e.g., Spanier $\mid 25$, p. 372, lines 1 to $3 \mid)$. Furthermore, if $i$ is the smallest integer with $\pi_{i}(P) \neq 0$, then by the Hurewicz Isomorphism Theorem (Spanier |25, 7.5.5., p. $398 \mid), \pi_{i}(P)$ is isomorphic to $H_{i}(P)$, the $i$ th singular homology group of $P$. Therefore, in particular, $\pi_{l}(P)$ is finitely generated, because $P$ has a finite number of cells in each dimension, being parafinite by assumption.

Being a finitely generated abelian group, $\pi_{I}(P)$ is therefore a direct sum of a finite number of infinite cyclical groups isomorphic to the integers, and of cyclical groups of finite prime order, these latter denoted by $Z_{p_{i}}, p_{i}$ prime numbers (Lederman 121, p. 92, p. 120, Definition 7, and p. 121, 
Proposition $17 \mid)$. The direct sum of the infinite cyclical groups generates the free part of $\pi_{i}(P)$; the direct sum of the rest its torsion $T\left(\pi_{i}(P)\right)$, where

$$
T\left(\pi_{l}(P)\right)=\left\{x \in \pi_{l}(P): m x=0 \text { for some integer } m \geqslant 1\right\} .
$$

Note also that $\left.\pi_{i}\left((P)^{k}\right)\right)$ is isomorphic to the product of $k$ copies of $\pi_{i}(P)$. i.e.,

$$
\pi_{i}\left((P)^{k}\right) \cong X_{j}^{k} \pi_{i}(P)
$$

(see, e.g., Spanier $\mid 25$, p. 419 , No. 5 of B $\mid$ ). Now, consider $x$ a generator in the free part of $\pi_{i}(P)$. Then $(x, x, \ldots, x) \in \pi_{i}\left((P)^{k}\right)$. Because of the condition of respect of unanimity,

$$
\phi^{*}(x, x, \ldots, x)=x
$$

Also, if $e$ is the identity element in the group $\pi_{i}(P)$, since $\phi^{*}$ is a group homomorphism, (i.e., $\phi^{*}$ is "linear" in the group operation denoted + ),

$$
\begin{aligned}
\phi^{*}(x, x, \ldots, x)= & \phi^{*}\{(x, e, \ldots, e) \dagger(e, x, e, \ldots, e) \dagger \ldots+(e, e, \ldots, e, x)\} \\
= & \phi^{*}(x, e, \ldots, e) \dagger \phi^{*}(e, x, \ldots, e, e) \dagger \ldots \\
& +\phi^{*}(e, e, \ldots, e, x)
\end{aligned}
$$

since $\quad(x, x, \ldots, x)=(x, e, \ldots, e) \dagger(e, x, e, \ldots, e) \dagger \ldots+(e, e, \ldots, e, x)$. By the symmetry condition, all factors in the last expression of (2) are identical, say, to $\phi^{*}(x, e, \ldots, e)$. It follows from (1) and (2) that

$$
x=k \phi^{*}(x, e, \ldots, e)=k y, \quad \text { where } \quad y=\phi^{*}(x, e, \ldots, e) \in \pi_{i}(P),
$$

because $\pi_{i}(P)$ is abelian. Since $x$ is a generator in the free part of $\pi_{i}(P), x$ generates a group which is isomorphic to the integers. Since $k$ is any arbitrarily chosen natural number, it follows that $x=0$, because only 0 can be divided by any integer $k$. This implies that all generators in the free part of $\pi_{i}(P)$ are zero, i.e., $\pi_{i}(P)$ has no free part.

Now consider $x$ a generator in the torsion part of $\pi_{i}(P), T\left(\pi_{i}(P)\right)$. Then $x \in Z_{p_{i}}$, for some $p_{1}$. However, from (3), $x=k y$, for any $k \in Z$. It follows that $Z_{p_{i}}$ is zero for all $p_{l}$, which implies that $T\left(\pi_{i}(P)\right)=0$ also. Since $\pi_{i}(P)$ has no torsion and no free part, it is the zero group, for all $i \geqslant 2$.

In the case $i=1$, note that when $\pi_{i}(P)$ is abelian, then the group $\pi_{1}(P)$ is isomorphic to $H_{1}(P)$ (see, e.g., Spanier $\mid 25$, remarks at top of p. 391|). It follows that when $\pi_{1}(P)$ is shown to be abelian, the rest of the proof above applies for the case $i=1$, since $H_{1}(P)$ is a finitely generated abelian group. Therefore we show next that the existence of the map $\phi$ implies immediately 
that $\pi_{1}(P)$ is abelian. Note that for any two elements $x$ and $y$ in $\pi_{1}(P)$, and for any $k \geqslant 1,(2)$ and (3) imply

$$
\begin{aligned}
x \dagger y & =k \phi^{*}(x, e, \ldots, e) \dagger k \phi^{*}(e, y, e, \ldots, e) \\
& =\phi^{*}(k x, k y, e, \ldots, e) \text { because } \phi^{*} \text { is a group homomorphism. }
\end{aligned}
$$

But by the symmetry condition on $\phi$,

$$
\phi^{*}(k x, k y, e, \ldots, e)=\phi^{*}(k y, k x, e, \ldots, e)=y \dagger x .
$$

Therefore (4) and (5) imply $x+y=y+x$, so that $\pi_{1}(P)$ is abelian. This completes the proof that $\pi_{i}(P)=0$ for all $i \geqslant 1$.

Since $P$ is a connected $C W$ complex and $\pi_{i}(P)=0$ for all $i \geqslant 1, P$ must be contractible (see, e.g., Spanier $\mid 25,7.6 .24$, p. $405 \mid$, and footnote 12 of this paper), completing the proof.

Proof of Corollary 1. Assume first that $L=X P$ admits $P$ as a deformation retract. It follows from Spanier $\mid 25$, p. 33, Theorem 13|, that $X P$ and $P$ have the same homotopy type. Hence

$$
\pi_{i}(P) \cong \pi_{i}(X P) \text { for } i \geqslant 1 .
$$

However, by Spanier $\mid 25$, p. 419 , No. 5 of $\mathrm{B} \mid$, there is an isomorphism

$$
\pi_{i}(X P) \cong \mathrm{X}_{j=1}^{k} \pi_{i}(P) \quad \text { for all } i \geqslant 0 .
$$

Therefore, for all $i \geqslant 1$

$$
\pi_{i}(P) \cong 0
$$

Since $\boldsymbol{P}$ is connected and is a $\boldsymbol{C W}$ complex this implies that $\boldsymbol{P}$ is contractible (see Spanier $\mid 25$, p. $405,7.6 .24 \mid$ and footnote 12 of this paper).

This completes the proof of sufficiency.

Assume now that $\boldsymbol{P}$ is contractible. It follows that there exists a deformation retract $R$ from $P$ into a fixed preference $p_{0}$ in $P$ (Spanier $[25$, p. 29|). Consider now the set

$$
Q=\left\{\left(p, p_{0}, \ldots, p_{0}\right): p \in P\right\}
$$

The deformation retract induced by $R$ on the last $k-1$ components of $X P$ maps $X P$ into $Q$. Since $Q$ is homeomorphic to $P$ this completes the proof of necessity. 


\section{RFFERENCES}

I. K. J. Arrow, "Social Choice and Individual Values," Yale Univ. Press, New Ilaven. Conn., 1951: 2nd ed., Wiley, New York, 1963.

2. C. Bissacia ani) A. Priczrynski, "Selected Topics in Infinite Dimensional Topology," Polska Akademia Nauk, Instyut Matema Tyczny, Warsaw, 1975.

3. D). Bı.ACK, On the rationale of group decision making. J. Pol. Eicon. 56 (1948), 2324.

4. D. Brown, Aggregation of preferences, Quart. J. Econ. 80 (1975), 456-469.

5. G. CuIIIII.NISky, Manifolds of preferences and equilibria, Report No. 27, Project on Ifficiency of Decision Making in Economic Systems, Harvard University, 1976.

6. G. Chicuin.nisky, The topological equivalence of the Pareto condition and the existence of a dictator, J. Math. Econ. 9 (1982), $223-233$.

7. G. CIIIIIII.NISky, Social aggregregation rules and continuity, Quart. J. Econ. 87 (1982).

8. G. Cincuit.nisky, "Single Peakedness and the Contractibility of Spaces of Preferences," working paper, University of Essex, 1980.

9. G. Cincilit.nisky, Social choice and the topology of spaces of preferences, Adr. in Math. 37. No. 2 (1980), 165-176.

10. G. Chicuin.nisky, The structural instability of decisive majority rules, J. Math. Econ. 9 (1982), 207-221.

II. G. Cincuit.nisky, "Intensity of Preferences and von Neumann-Morgenstern Utilities," Mathematics of Operations Research, in press.

12. G. Cincilil nisky anI) G. M. Heal, Community preferences and social choice, Fissex Economic Papers No. 165, J. Math. Econ., 1983.

13. G. Cinchinnisky AND G. M. HeAl, "Social Choice with Infinite Populations: Construction of a Social Choice Rule and Impossibility Results," Columbia University Economics Discussion Paper, 1979.

14. J. Cilipman, Homothetic preferences and aggregation, J. Econ. Theory 8 (1974), 26-38.

15. P. Coti(ill IN ANI) K. P. LIN, Continuity properties of majority rule with intermediate preferences, Mathematical Social Sciences I (1981), 289-296.

16. G. Diнк1:U, Smooth preferences, Econometrica 40 (1972), 603-615.

17. P. C. Fisılaurn, Arrow impossibility theorem: Concise proof and infinite voters, J. Econ. Theory 2 (1970), 103-106.

18. G. M. Hr:Al., Contractibility and public decision making, in "Social Choice and Welfare" (P. K. Pattanaik and M. Salles, Eds.), North Holland, Amsterdam, 1983.

19. A. P. Kirman ani) D. Sonderman, Arrow's theorem: Many agents and invisible dictators, J. Econ. Theory 5 (1972), 267-277.

20. (i. H. Кялмі: , On a class of equilibrium conditions for majority rule, Econometrica (1972).

21. N. II. Kuiper, "Varietes hilbertiennes, aspects geometriques," Seminaire de Mathematiques Superieures, Le Presses de I'Universite de Montreal, Montreal, 1971.

22. W. Li:1):kmAN, "Introduction to Group Theory," Oliver \& Boyd, Edinhurgh, 1973.

23. (. F. MAUni): R, "Algebraic Topology," Van Nostrand-Reinhold, London, 1970.

24. A. K. SIN ANII P. K. PATtANAIK, Necessary and sufficient conditions for rational choice under majority decision, J. Econ. Theory I (1969), 178-202.

25. I:. Sipnil:k, "Algebraic Topology," McGraw-Hill Series in Higher Mathematics, McGraw Hill, New York, 1966. 